\title{
LETTERS-CONCISE RESEARCH REPORTS Association of Pharmaceutical Manufacturer Payments to Physicians and Prescribing Dosage of Opioids
}

\author{
William Fleischman, MD, MHS ${ }^{1,2}$, Shantanu Agrawal, MD, MPhil,3, Cary P. Gross, MD ${ }^{4,5}$, \\ and Joseph S. Ross, MD, MHS $4,5,6$
}

\begin{abstract}
'Department of Patient Safety \& Quality, Hackensack Meridian Health, Edison, NJ, USA; ${ }^{2}$ Centers for Medicare \& Medicaid Services, Baltimore, MD, USA; ${ }^{3}$ National Quality Forum, Washington, DC, USA; ${ }^{4}$ Section of General Internal Medicine, Department of Medicine, Yale School of Medicine, New Haven, CT, USA; ${ }^{5}$ Department of Health Policy and Management, Yale School of Public Health, New Haven, CT, USA; ${ }^{6}$ Center for Outcomes Research and Evaluation, Yale-New Haven Health, New Haven, CT, USA.
\end{abstract}

$\mathrm{J}$ Gen Intern Med 34(7):1074-6

DOI: $10.1007 / \mathrm{s} 11606-019-04897-9$

(c) Society of General Internal Medicine (This is a U.S. government work and not under copyright protection in the U.S.; foreign copyright protection may apply) 2019

\section{BACKGROUND AND OBJECTIVE}

Pharmaceutical manufacturers spend billions of dollars annually on payments to physicians in the form of food, gifts, educational materials, and speaker and consulting fees. Payments related to brand-name cardiovascular and diabetes drugs have recently been associated with higher rates of prescribing, despite lower-cost alternatives being available. ${ }^{1-3}$ Given the growing misuse of prescription opioids and accompanying public health risks, we used Centers for Medicare \& Medicaid Services Open Payments data, along with Medicare Part D prescribing data, to examine associations between opioid-related payments from pharmaceutical manufacturers to physicians and opioid prescribing. We assessed both average prescribed dose and whether the prescribed dose exceeded Centers for Disease Control and Prevention (CDC) opioid dosage recommendations. ${ }^{4}$

\section{METHODS}

We included opioid-related non-research payments from pharmaceutical manufacturers to doctors of medicine, osteopathy, dentistry, optometry, and chiropractic from August 2013 through December 2015, a time period for which we had access to both payment and prescribing data. Using provider identifiers, we linked payments to Medicare Part D prescription claims for opioids over the same period, excluding prescriptions for methadone and buprenorphine, and for patients with hospice claims or diagnoses of cancer during the study period.

Recent CDC chronic pain opioid prescribing guidelines recommend physicians use caution when prescribing dosages $\geq 50 \mathrm{mg}$ morphine equivalents per day (MME/day) and to avoid dosages $\geq 90 \mathrm{MME} /$ day. ${ }^{4}$ We used these thresholds as

Published online April 22, 2019 markers of high- or low-dose prescribing (though not to assess guideline compliance, as available prescription data was from prior to guideline publication). Because patients frequently received opioid prescriptions from multiple physicians, the individual patient-individual physician cluster was our unit of analysis. For each patient-physician cluster, we calculated $\mathrm{MME} /$ day by dividing the total dosage prescribed by the total days of drug supplied. Our primary outcome was the binary measure of whether a patient's opioid prescriptions by a physician were $\geq 90 \mathrm{MME} /$ day, while $\geq 50 \mathrm{MME} /$ day and mean daily MME were secondary outcomes.

We performed descriptive analysis, then used multiple logistic regression to examine associations between opioidrelated payments and prescribing of both $\geq 90$ and $\geq 50$ MME/day, overall and stratified by physician specialty categories. We then examined associations between opioid-related payments and mean daily MME prescribed using generalized linear mixed models using a $2 \%$ random sample of beneficiaries, accounting for clustering among physicians and patients. Analyses were adjusted for physician specialty, patient age, sex, race, dual-eligibility status, Medicare copayment status, and clinical comorbidity using the Hierarchical Condition Category score. Analysis was conducted using Stata and R. The Yale University Institutional Review Board exempted the study from review.

\section{FINDINGS}

During the study period, there were 416,678 opioid-related payments from pharmaceutical manufacturers to 63,941 physicians (Table 1). The median payment value was $\$ 13$ (interquartile range 10-17). In addition, there were 141,237,664 prescriptions for opioids to $17,808,859$ beneficiaries by 750,063 physicians. Approximately $45 \%$ of Part D beneficiaries received opioid prescriptions; $23 \%$ filled prescriptions averaging 50-89 MME/day, and 7\% $\geq 90 \mathrm{MME} /$ day. Opioid-related payments were associated with a higher likelihood of exceeding prescribed dosages of $90 \mathrm{MME} /$ day $(\mathrm{OR}=1.27,95 \% \mathrm{CI}, 1.25-1.30, p<0.001)$ and $50 \mathrm{MME} /$ day $(\mathrm{OR}=1.14,95 \% \mathrm{CI}, 1.12-1.15, p<0.001)$, overall and for nearly all physician specialty categories (Table 2 ), as well 
Table 1 Characteristics of opioid-related payments from manufacturers to physicians, by most common drugs associated with payments and by payment types, August 2013-December 2015

\begin{tabular}{llll}
\hline \hline & No. of payments & Sum paid, \$ & Median payment, \$ (interquartile range) \\
\hline Opioid attributed to payment & & & \\
Oxycontin (oxycodone) & 133,403 & $2,896,460$ & $13.3(11-16)$ \\
Hysingla ER (hydrocodone) & 65,404 & $5,769,877$ & $13.7(11-18)$ \\
Subsys (fentanyl) & 62,359 & $16,339,192$ & $13.9(8-58)$ \\
Embeda (morphine/naltrexone) & 52,230 & $1,590,898$ & $10.3(4-13)$ \\
Nucynta (tapentadol) & 47,127 & $4,275,864$ & $13.7(10-19)$ \\
Xartemis (oxycodone-acetaminophen) & 36,076 & $1,228,933$ & $13.5(11-17)$ \\
Fentora (fentanyl citrate) & 20,413 & $1,228,933$ & $13.9(10-19)$ \\
Zohydro ER (hydrocodone) & 18,688 & $1,097,209$ & $12.3(10-16)$ \\
Exalgo (hydromorphone) & 12,079 & 375,984 & $12.8(10-16)$ \\
Opana (oxymorphone) & 5472 & 115,756 & $14.2(12-18)$ \\
All others & 9030 & $1,429,319$ & $17.3(13-28)$ \\
Totals* & 416,678 & $36,271,638$ & $13.2(10-17)$ \\
& & & \\
Payment type & 389,460 & $6,915,500$ & $12.9(10-16)$ \\
Food and beverage & 12,206 & $22,419,327$ & $1900(839-2400)$ \\
Speaker fees & 6217 & 112,498 & $7.2(3-18)$ \\
Journal articles/textbooks & 6804 & $2,433,233$ & $160(63-479)$ \\
Travel and lodging & 812 & $2,107,618$ & $2500(990-3080)$ \\
Consulting fees & 1029 & $2,222,409$ & $1800(1300-2300)$ \\
Honoraria & 144 & 16,054 & $67.5(13-100)$ \\
Gifts & 6 & 45,000 & $7500(7500-7500)$ \\
Grants & 416,678 & $36,271,638$ & $13.2(10-17)$ \\
Totals &
\end{tabular}

*Each payment can be associated with up to 5 drugs; hence, the number of individual payments attributed to opioids and their sum value exceed the total number of payments and sum value

as with a $4.5 \%$ higher mean daily MME (95\% CI, 4.0-5.0\%, $p<0.001)$.

\section{DISCUSSION}

Our analysis demonstrates that opioid-related payments to physicians from pharmaceutical manufacturers were associated with greater likelihood of Medicare beneficiaries without claims for hospice or diagnoses of cancer being prescribed opioids at higher dosages. Our findings build on a recent analysis suggesting pharmaceutical manufacturer payments related to opioids may influence opioid prescribing, ${ }^{5}$ and with prior research demonstrating associations between industry payments and physician prescribing. ${ }^{1-3}$ The median payment value of $\$ 13$ is also consistent with prior Open Payments analyses ${ }^{3,5,6}$ and points to the potential influence of even small payments.

Our study is limited by its cross-sectional design, demonstrating only correlations between payments and prescribing. It is possible, for example, that manufacturers market to physicians who already prescribe higher opioid doses. In addition, we may underestimate opioid use as Medicare beneficiaries may pay for prescriptions out-of-pocket, particularly those available as generics. Nevertheless, our findings highlight the influence and potential patient and public health risks that may be associated with industry payments to physicians, such as higher-risk opioid prescribing.

Table 2 Associations between opioid-related payments and physician-Medicare beneficiary aggregate prescribing of both $\geq 50$ and $\geq 90 \mathrm{MME} /$ day, overall and stratified by physician type and specialty group, August 2013-December 2015

\begin{tabular}{llr}
\hline \hline & \multicolumn{1}{c}{ Odds ratio (95\% CI) } \\
\cline { 2 - 3 } & $\mathbf{2 5 0}$ MME/day & $\geq \mathbf{9 0}$ MME/day \\
\hline All physicians & $1.14(1.12-1.15)$ & $1.27(1.25-1.30)$ \\
By physician category & & \\
Family/general practice, internal medicine & & $1.31(1.28-1.33)$ \\
Emergency medicine & $1.14(1.12-1.16)$ & $4.00(3.07-5.20)$ \\
Pain medicine, anesthesia, psychiatry, neurology & $1.28(1.08-1.50)$ & $1.57(1.48-1.67)$ \\
Physical, neuromusculoskeletal and sports medicine & $1.71(1.61-1.81)$ & $1.53(1.42-1.66)$ \\
Surgical specialties & $1.38(1.29-1.47)$ & $1.69(1.63-1.76)$ \\
Dentists & $1.05(1.03-1.08)$ & $1.65(1.11-2.47)$ \\
Chiropractors & $1.55(1.26-1.91)$ & $1.22(0.27-5.45)$ \\
All other specialties & $0.91(0.25-3.33)$ & $1.97(1.72-2.26)$ \\
\hline
\end{tabular}

Logistic regression models were adjusted for prescriber specialty, patient age, sex, race, Medicare copay status, and fee-for-service status and for clinical comorbidity using the Hierarchical Condition Category risk score. The 95\% confidence intervals account for clustering among prescribers 
Corresponding Author: William Fleischman, MD, MHS; Department of Patient Safety \& QualityHackensack Meridian Health, Edison, NJ, USA (e-mail: wf3@buffalo.edu).

Authors' Contributions WF and JSR conceived and designed the study, assisted by all authors. WF acquired the data and performed analyses and interpretation with the assistance of all authors. WF drafted the manuscript and all authors critically revised it. JSR supervised the study.

Data Availability The datasets generated or analyzed as part of this study are not available due to restrictions on the use and sharing of Medicare beneficiary and prescribing data. The payments data are publicly available at https://www.cms.gov/openpayments/. Statistical code used to generate results is available from the corresponding author upon request.

\section{Compliance with Ethical Standards:}

Conflict of Interest: At the time of writing, Drs. Fleischman and Agrawal were employees of the Centers for Medicare \& Medicaid Services, which administers the Open Payments program. Drs. Gross and Ross receive support through Yale University from Johnson and Johnson to develop methods of clinical trial data sharing. Dr. Gross receives funding from 21st Century Oncology and the National Comprehensive Cancer Center-Pfizer program for research. Dr. Ross receives support through Yale University from the Centers of Medicare and Medicaid Services to develop and maintain performance measures that are used for public reporting, from Medtronic, Inc. and the Food and Drug Administration (FDA) to develop methods for post-market surveillance of medical devices, from the Food and Drug Administration as part of the Centers for Excellence in Regulatory Science and Innovation (CERSI) program, from the Blue Cross Blue Shield Association to better understand medical technology evaluation, and from the Laura and John Arnold Foundation to support the Collaboration on Research Integrity and Transparency (CRIT) at Yale.
Disclaimer: The views expressed in this article are those of the authors and do not necessarily represent the views of the Centers for Medicaid \& Medicare Services, the Department of Health and Human Services, or the United States Government.

\section{REFERENCES}

1. Yeh JS, Franklin JM, Avorn J, Landon J, Kesselheim AS. Association of industry payments to physicians with the prescribing of brand-name statins in Massachusetts. JAMA Intern Med. 2016;176(6):763-768. doi:https://doi.org/10.1001/jamainternmed.2016.1709

2. DeJong C, Aguilar T, Tseng C, Lin GA, Boscardin W, Dudley $\mathbf{R}$. Pharmaceutical industry-sponsored meals and physician prescribing patterns for Medicare beneficiaries. JAMA Intern Med. 2016;176(8):11141122. doi:https://doi.org/10.1001/jamainternmed.2016.2765

3. Fleischman W, Agrawal S, King $\mathbf{M}$, et al. Association between payments from manufacturers of pharmaceuticals to physicians and regional prescribing: cross sectional ecological study. BMJ. 2016;354:i4189. doi:https://doi.org/10.1136/bmj.i4189

4. CDC Guideline for Prescribing Opioids for Chronic Pain - United States, 2016. MMWR Recomm Rep. 2016;65. https://doi.org/10.15585/mmwr. rr650leler

5. Hadland SE, Cerdá M, Li Y, Krieger MS, Marshall BDL. Association of Pharmaceutical Industry Marketing of Opioid Products to Physicians With Subsequent Opioid Prescribing. JAMA Intern Med. 2018;178(6):861-863. doi:https://doi.org/10.1001/jamainternmed.2018.1999

6. Fleischman W, Ross JS, Melnick ER, Newman DH, Venkatesh AK. Financial Ties Between Emergency Physicians and Industry: Insights From Open Payments Data. Ann Emerg Med. 2016;68(2):153-158. doi:https:// doi.org/10.1016/j.annemergmed.2016.01.014

Publisher's Note: Springer Nature remains neutral with regard to jurisdictional claims in published maps and institutional affiliations. 